\title{
BMJ Open Supporting care for suboptimally controlled type 2 diabetes mellitus in general practice with a clinical decision support system: a mixed methods pilot cluster randomised trial
}

\author{
Mark E Murphy (D) , ${ }^{1}$ Jenny McSharry, ${ }^{2}$ Molly Byrne, ${ }^{2}$ Fiona Boland (D) , \\ Derek Corrigan, ${ }^{1}$ Paddy Gillespie, ${ }^{3}$ Tom Fahey (D) , ${ }^{1}$ Susan M Smith ${ }^{1}$
}

To cite: Murphy ME, McSharry J, Byrne M, et al. Supporting care for suboptimally controlled type 2 diabetes mellitus in general practice with a clinical decision support system: a mixed methods pilot cluster randomised trial. BMJ Open 2020;10:e032594. doi:10.1136/ bmjopen-2019-032594

- Prepublication history and additional material for this paper are available online. To view these files, please visit the journal online (http://dx.doi. org/10.1136/bmjopen-2019032594).

Received 26 June 2019 Revised 22 November 2019 Accepted 16 December 2019

\section{Check for updates}

(C) Author(s) (or their employer(s)) 2020. Re-use permitted under CC BY-NC. No commercial re-use. See rights and permissions. Published by BMJ.

${ }^{1}$ Department of General Practice, HRB Centre for Primary Care Research, Royal College of Surgeons, Dublin, Ireland

${ }^{2}$ Health Behaviour Change Research Group, School of Psycology, NUI Galway, Galway, Ireland

${ }^{3}$ School of Business and Economics, National University of Ireland, Galway, Ireland

Correspondence to Dr Mark E Murphy; markmurphy@rcsi.ie

\section{ABSTRACT}

Objectives We developed a complex intervention called DECIDE (ComputeriseD dECislonal support for suboptimally controlleD typE 2 Diabetes mellitus in Irish General Practice) which used a clinical decision support system to address clinical inertia and support general practitioner (GP) intensification of treatment for adults with suboptimally controlled type2 diabetes mellitus (T2DM). The current study explored the feasibility and potential impact of DECIDE.

Design A pilot cluster randomised controlled trial. Setting Conducted in 14 practices in Irish General Practice.

Participants The DECIDE intervention was targeted at GPs. They applied DECIDE to patients with suboptimally controlled T2DM, defined as a glycated haemoglobin (HbA1c) $\geq 70 \mathrm{mmol} / \mathrm{mol}$ and/or blood pressure $\geq 150 / 95 \mathrm{mmHg}$.

Intervention The intervention incorporated training and a web-based clinical decision support system which supported; (i) medication intensification actions; and (ii) non-pharmacological actions to support care. Control practices delivered usual care.

Primary and secondary outcome measures Feasibility and acceptability was determined using thematic analysis of semi-structured interviews with GPs, combined with data from the DECIDE website. Clinical outcomes included $\mathrm{HbA1c}$, medication intensification, blood pressure and lipids.

Results We recruited 14 practices and 134 patients. At 4-month follow-up, all practices and 114 patients were followed up. GPs reported finding decision support helpful navigating increasingly complex medication algorithms. However, the majority of GPs believed that the target patient group had poor engagement with GP and hospital services for a range of reasons. At follow-up, there was no difference in glycaemic control $(-3.6 \mathrm{mmol} /$ $\mathrm{mol}(95 \% \mathrm{Cl}-11.2$ to 4.0$))$ between intervention and control groups or in secondary outcomes including, blood pressure, total cholesterol, medication intensification or utilisation of services. Continuation criteria supported proceeding to a definitive randomised trial with some modifications.

\section{Strengths and limitations of this study}

- The DECIDE pilot cluster randomised controlled trial (RCT) assessed the feasibility of a complex intervention which sought to support general practitioner (GP)-based care for patients with suboptimally controlled type 2 diabetes mellitus (T2DM).

- Clinical inertia in the form of appropriate intensification of medications for adults with T2DM is a recognised problem from observational studies; this pilot RCT suggests that GPs apply individualised targets to many patients with suboptimal control of glycaemic and cardiovascular risk factors, but that a reluctance to intensify medication to meet these targets persists.

- DECIDE was found to be feasible and acceptable to GPs, though GPs reported that problems with patient engagement for a wide variety of reasons also led to suboptimal control of diabetes.

- Wider impacts on glycaemic and blood pressure control need to be considered and tested through a definitive randomised trial, addressing nonengagement, psychosocial factors and integration of software into the electronic health record for the purposes of intensification of medication.

Conclusion The DECIDE study was feasible and acceptable to GPs but wider impacts on glycaemic and blood pressure control need to be considered for this patient population going forward.

Trial registration number ISRCTN69498919

\section{INTRODUCTION}

Suboptimal control of blood sugar and cardiovascular risk factors in type 2 diabetes mellitus (T2DM) results in higher morbidity, poorer quality of life and increased healthcare costs. ${ }^{12}$ Despite this, many patients continue to have poor control for several years before intensification with medications. ${ }^{3-6}$ We conducted a systematic review of interventions targeting 
patients with a glycatedhaemoglobin (HbA1c) over $59 \mathrm{mmol} / \mathrm{mol}(7.5 \%)$ and found that interventions delivered only modest benefits, though meta-regression analysis suggested that interventions targeting patients with higher baseline $\mathrm{HbAlc}$ levels were associated with greater improvements in glycaemic control. ${ }^{7}$

Suboptimal control of T2DM can be influenced by patient and physician factors. Patient influences include reduced medication adherence, lack of engagement with healthcare services and psychosocial difficulties. ${ }^{8}$ Physician factors may relate to a failure to intensify evidencebased treatments, referred to as clinical inertia. ${ }^{9-11}$ Clinical inertia has been reported in different countries, through all stages of intensification pathways. ${ }^{32-18}$ Clinical inertia could be addressed through the use of clinical decision support systems (CDSSs). ${ }^{18-20}$ CDSSs involve computer software designed to support decision-making, matching individual patient characteristics to a computerised clinical knowledge base and then providing patient-specific assessments or recommendations to support a decision that can relate to diagnosis, investigation, prognosis or treatment. ${ }^{21}$ T2DM decision-making has become more complex, which may have an impact on treatment escalation, and CDSS could support this decision-making. ${ }^{22-24}$

DECIDE (ComputeriseD dECisIonal support for suboptimally controlleD typE 2 Diabetes mellitus in Irish General Practice) is a complex intervention that was developed to address clinical inertia and medication intensification. ${ }^{25}$ The first stage of the UK Medical Research Council (MRC) framework was used, in conjunction with the behaviour change wheel (BCW) as a theoretical guide to intervention development. ${ }^{26-28}$ The process of intervention development, ${ }^{25}$ the results of the non-randomised pilot study ${ }^{29}$ and the protocol for the DECIDE intervention have been reported previously ${ }^{29}$ (online supplementary file 1). We aimed to examine the feasibility and potential clinical impact of the DECIDE intervention through a pilot cluster randomised control trial (RCT).

\section{METHODS}

\section{Design and setting}

A pilot cluster RCT was conducted in Irish General Practice (box 1). The Consolidated Standards of Reporting Trials statement, extended for use in cluster RCTs, was used to conduct and report the study (online supplementary file 2). ${ }^{30}$

Research Ethics Committee; GPs, the representatives of each cluster, were consented for the study.

\section{Patient and public involvement}

There were no funds or time allocated for patientand public involvement, so we were unable to involve patients in the development of this study. This research was done without patient involvement. Patients were not invited to comment on the study design and were not consulted to develop patient relevant outcomes or interpret the results.
Box 1 Access to healthcare and structure of type2 diabetes mellitus (T2DM) care in the Ireland

Access to General Practice healthcare in the Ireland

- The General Medical Services (GMS) scheme provides medical care to approximately $34 \%$ of the Irish population. It is predominantly means-tested and provides those who are eligible with free general practitioner visits, free hospital care and free medications (except for a prescription levy, currently $€ 2.50$ per item to a maximum of $€ 25)$. A further $\sim 10 \%$ of the population are entitled to free doctor visits (called a Doctor Visit Card (DVC)) based on means testing and age-banding (all Under-6-year-olds and Over-70-year-olds).

- The Long Term IIIness (LTI) Scheme allows persons with certain medical conditions (T2DM being one) to have free access to medications which treat that condition. All patients with T2DM can avail of free medications under the LTI Scheme.

- The GMS and LTI schemes are administered by the Health Services Executive and Primary Care Reimbursement Services.

- 'Private patients' represent approximately $45 \%$ of the population and are not entitled to a GMS or DVC card, paying the full cost for attending a generalpractitioner (GP), out-of-pocket, at the point of healthcare delivery.

\section{Structure of diabetes care in Ireland}

- Before October 2015, structured chronic disease management of T2DM was not universally available in Irish primary care. Approximately 10 primary care schemes existed in 2013 and 2014, providing different levels of structured T2DM care, often set up as pilot schemes. This represented a maximum of 250 practices within Irish general practice (approximately $10 \%$ of total practices). Up until October 2015, the vast majority of structured T2DM care in Ireland was provided in secondary care, through public hospital outpatients or under the care of endocrinologists in private clinics.

- In October 2015, a new agreement was reached with GPs entitling all GMS patients to a structured diabetes programme in primary care (called a Diabetes Cycle of Care) with two free GP visits per annum. Private patients with T2DM either pay to receive care from their GP or continue to attend secondary care.

Patients were not invited to contribute to the writing or editing of this document for readability or accuracy.

\section{Recruitment, eligibility and randomisation}

GPs participating in four research and diabetes interest groups in Ireland were invited to participate by email. GPs and practices were eligible for inclusion if they used either of the most common electronic health record (EHR) systems in Ireland. Patients were included in the study - and GPs applied the intervention to them - if patients had:

- 'Suboptimal or poor control' of T2DM: There is no valid cut-off which defines the term 'poor control' and as a linguistic phrase it is argued it can demotivate, induce fear, guilt or distress and should be avoided. ${ }^{31}$ Patients with T2DM were included if they had suboptimal control, which we defined as significant hyperglycaemia (HbAlc $\geq 70 \mathrm{mmol} / \mathrm{mol}$ ) and/ or if they had significant elevation of blood pressure (BP) $(\geq 150 / 95 \mathrm{~mm} \mathrm{Hg})$.

- Age between 18 to 75 years. 
Targeting cardiovascular risk factors in T2DM, through the control of BP and lipids, is known to improve cardiovascular morbidity and mortality, ${ }^{32}$ however intensive reductions in blood pressure ${ }^{33}$ and lipids ${ }^{34}$ has less evidence. Therefore significant elevation of BP (>150/95 mm Hg) was included in our inclusion criteria of 'poor control'. While the impact of glucose lowering in T2DM has been demonstrated for microvascular complications, it has conflicting evidence on cardiovascular outcomes. ${ }^{35-38}$ Despite this, glucose lowering recommendations for microvascular and macrovascular complications are present in both Irish College of General Practitioners and National Institute for Clinical Excellence clinical guidelines. ${ }^{2324}$ Therefore we included significant elevation of both blood pressure and HBA1c in our inclusion criteria; (a) as they are both important variables which can mediate cardiovascular morbidity if they are significantly elevated; and (b) as evidence-based guidelines recommend that clinicians address their management, if elevated, through non-pharmacological actions and pharmacological intensification. ${ }^{23}{ }^{24}$ Patients with type 1 diabetes mellitus (T1DM) were excluded. Discrepancies between the original trial registration and the pilot cluster RCT, including the collection of outcomes and follow-up length are outlined in online supplementary file 3.

An electronic health record 'finder tool' was developed by the study team over 2016 to 2018 with our project collaborators to enable GPs create a list of T2DM patients which met the inclusion criteria of suboptimal hyperglycaemia and elevated BP. This list of patients included three categories of patients with T2DM for inclusion; (a) HbA1c $\geq 70 \mathrm{mmol}$ and elevated BP (systolic blood pressure (SBP) $\geq 150 \mathrm{~mm} \mathrm{Hg}$ or diastolic blood pressure (DBP) $\geq 95 \mathrm{~mm}$ $\mathrm{Hg}$ ), (b) HbAlc $\geq 70 \mathrm{mmol}$ (and $\mathrm{BP}<150 / 95 \mathrm{~mm} \mathrm{Hg}$ ) and (c) SBP $\geq 150 \mathrm{~mm} \mathrm{Hg}$ or DBP $\geq 95 \mathrm{~mm} \mathrm{Hg}$ (but HbAlc $<70 \mathrm{mmol} / \mathrm{mol}$ ). GPs generated a list of eligible patients and from this list, 10 patients were selected for inclusion by the study statistician (FB), by computer-generated stratified-randomisation, initially including those patients with suboptimal control of HbAlc and BP initially, then HbA1c alone, then BP alone.

The study statistician allocated practices to intervention or control by minimisation. ${ }^{39}$ Minimisation factors included practice size and previous enrolment in a structured diabetes care service. GPs were not blinded due to the nature of the intervention. Practices were allocated before baseline data collection as different data was collected for each group. All practices entered baseline data between October 2018 and December 2018. At this point, intervention practices were invited to use the DECIDE CDSS.

\section{Intervention}

Details of the DECIDE intervention, including a description of content in terms of behaviour change techniques, ${ }^{40}$ have been outlined previously. ${ }^{25}{ }^{29}$ In brief, the components of the DECIDE intervention included: a. GP Training: A training video on the DECIDE CDSS and an educational folder with guidelines on medication intensification information. ${ }^{23} 24$

b. DECIDE CDSS and website which included:

- Entry of anonymised patient-specific data.

- Treatment algorithms for medications relating to glycaemic, hypertension and lipid control. CDSS options were triggered by baseline patient information, matched through decision trees in the DECIDE website and delivering tailored recommendations to the GP.

- Suggestions for non-pharmacological actions.

- Free-text boxes for GPs to record why they chose not to intensify treatment with.

The CDSS prompted GPs to consider treatment escalation, through a shared decision model, for T2DM patients with significant elevation of HbAlc and blood pressure. The intervention period lasted for 1 month after randomisation. A more detailed description of the intervention, through the Template for Intervention Description and Replication checklist is reported in online supplementary file 4 and a copy of the educational material is outlined in online supplementary file $5 .{ }^{41}$

\section{Control}

Control practices delivered usual care (see box 1). Access to the CDSS-pharmacological intensification options and non-pharmacological options were not available to control practices.

\section{Outcomes and data collection}

As this was a pilot study, the primary outcomes related to feasibility of the intervention and also considered impact on glycaemic control (HbA1c). In comparison to the trial registration (online supplementary file 3 ), HbAlc was not the primary outcome, but was used to estimate a potential sample size. Secondary clinical outcomes included changes in SBP and DBP, total cholesterol, intensification of glycaemic, blood pressure and lipid-lowering medications and measures of healthcare utilisation. Clinical outcomes were entered by GPs and practice nurses and collected through the DECIDE website. Outcomes were collected at 4 months following baseline data insertion.

\section{Feasibility and acceptability}

Feasibility and acceptability of DECIDE was assessed using predefined continuation criteria (see table 1$){ }^{42}$

Semi-structured interviews were conducted with intervention GPs, by telephone (MEM) and were audio recorded. A topic guide (see online supplementary file 6) was created, which included questions relating to intervention fidelity, continuation criteria and participant experiences. Interviews were transcribed and coded by MEM using a directed qualitative content analysis approach ${ }^{43}$ informed by the continuation criteria for the study to generate initial themes. Three transcripts were coded by a second author, JM who then met with MEM to 
Table 1 Continuation criteria, agreed prior to the commencement of the DECIDE pilot cluster RCT

\section{Proceed with RCT}

Recruitment of GPs Recruitment of 14 general practices within 3 months

Retention of GPs

Retention of $\geq 14$ general practices throughout 4-month intervention period

Recruitment of patients

Retention of patients Intervention feasibility and acceptability

Outcomes
Recruitment of 140 study patients across all practices

Retention of $\geq 90 \%$ study patients for follow-up

DECIDE intervention acceptable to $\geq 75 \%$ GPs, intervention personnel and patients involved Intervention identifies outcome measures which are appropriate and acceptable to stakeholders and have a positive effect on patients

Intervention demonstrates potential cost-effectiveness; that is, through cost savings which are likely to outweigh the direct cost of the intervention, or through additional costs which are likely to be deemed acceptable given the potential health outcome gains
Proceed with RCT following some changes to the protocol

Recruitment of 12 general practices Unable to recruit at least 12 within 3 months

Retention of $\geq 12$ general practices throughout 4-month intervention period general practices within 3 months

Retention of $<12$ general practices throughout 4-month intervention period

Recruitment of $\geq 112$ study patients across all practices

Recruitment of $<111$ study patients across all practices

Retention of $\geq 80 \%$ study patients for follow-up

Retention of $<80 \%$ study patient for follow-up

DECIDE intervention acceptable to $\geq 50 \%$ GPs, intervention personnel and patients involved

Intervention identifies some outcome measures which are appropriate and acceptable to stakeholders and have some positive effects on patients though further refinement of outcome measures needed

Intervention demonstrates potential Intervention does not cost effectiveness; that is, through demonstrate potential cost some cost savings which may outweigh the direct cost of the intervention, or through additional costs which are likely to be deemed acceptable given the potential health outcome gains
DECIDE intervention acceptable to $<50 \%$ GPs, intervention personnel and patients involved

DECIDE intervention does not identify outcome measures which are appropriate effectiveness

DECIDE, ComputeriseD dECislonal support for suboptimally controlleD typE 2 Diabetes mellitus in Irish General Practice; GPs, general practitioners; RCT, randomised controlled trial.

further refine themes and subthemes. Final themes were then discussed with the study team.

DECIDE website activity captured GPs engagement with the CDSS treatment algorithms and their use of the website, with quantitative measures of feasibility and freetext responses.

\section{Sample size}

A formal sample size calculation was not required for a pilot RCT. ${ }^{30}$ However, based on an average baseline HbAlc of $91.1 \mathrm{mmol} / \mathrm{mol}$ (SD 16.7) and an intracluster correlation of 0.027 we estimated 14 practices, each with 10 patients, would give us $80 \%$ power to detect a clinically meaningful reduction of $10 \mathrm{mmol} / \mathrm{mol}$ in $\mathrm{HbAlc}$ and includes a $10 \%$ loss to follow-up.

\section{Analysis of clinical outcomes}

Descriptive statistics were used to describe practice and patient characteristics. GP survey data and data collected from the DECIDE website provided recruitment and attrition rates. For HbA1c, SBP, DBP and total cholesterol we conducted an intention-to-treat analysis including all randomised participants, all retained in the group to which they were allocated and using last observation carried forward for missing values. ${ }^{45}$ Furthermore, we conducted a per protocol (PP) analysis which excluded those who did not adequately adhere to the protocol and those with missing outcome data. All analyses used appropriate regression models, linear (HbAlc, SBP, DBP and total cholesterol), poisson (utilisation of services measures) or logistic (intensification of medication (Yes/No)) and included a random practice effect to account for the correlation between patients in practices. A second model was performed for HbAlc, BP and cholesterol to factor if a lag time, which we called recency of testing, from measurement to baseline information insertion (168 days) and follow-up collection (60 days). A third model was performed for HbAlc to adjust for insulin use at baseline. Furthermore, for HbAlc, subgroup analyses based on age $(<65$ and $\geq 65)$ and gender) were performed as outlined in the protocol. Stata V.15 was used for all analyses. ${ }^{46}$ Stata V.15 was used for all analyses, specifically the commands mixed, mepoisson and melogit. 


\section{Pilot health economic evaluation}

Healthcare resource items were identified and collected, to inform the cost component of a future health economic evaluation, and included GP visits, nurse visits, medications, hospital inpatient admissions, outpatient department visits and emergency departments visits.

\section{RESULTS}

The continuation criteria for the study were used to structure the results, relating to recruitment and retention, feasibility and acceptability and quantitative clinical outcome analysis.

\section{Recruitment and retention}

In total, 134 patients from 14 practices were recruited, 67 patients in each arm. Figure 1 shows the flow of participants through the trial. The target number for recruitment in each practice was 10 patients. The finder tool found a mean of 21 patients per practice meeting the inclusion criteria overall, but 6 of the 14 practices, only 9 patients met the inclusion criteria. Overall the mean number of

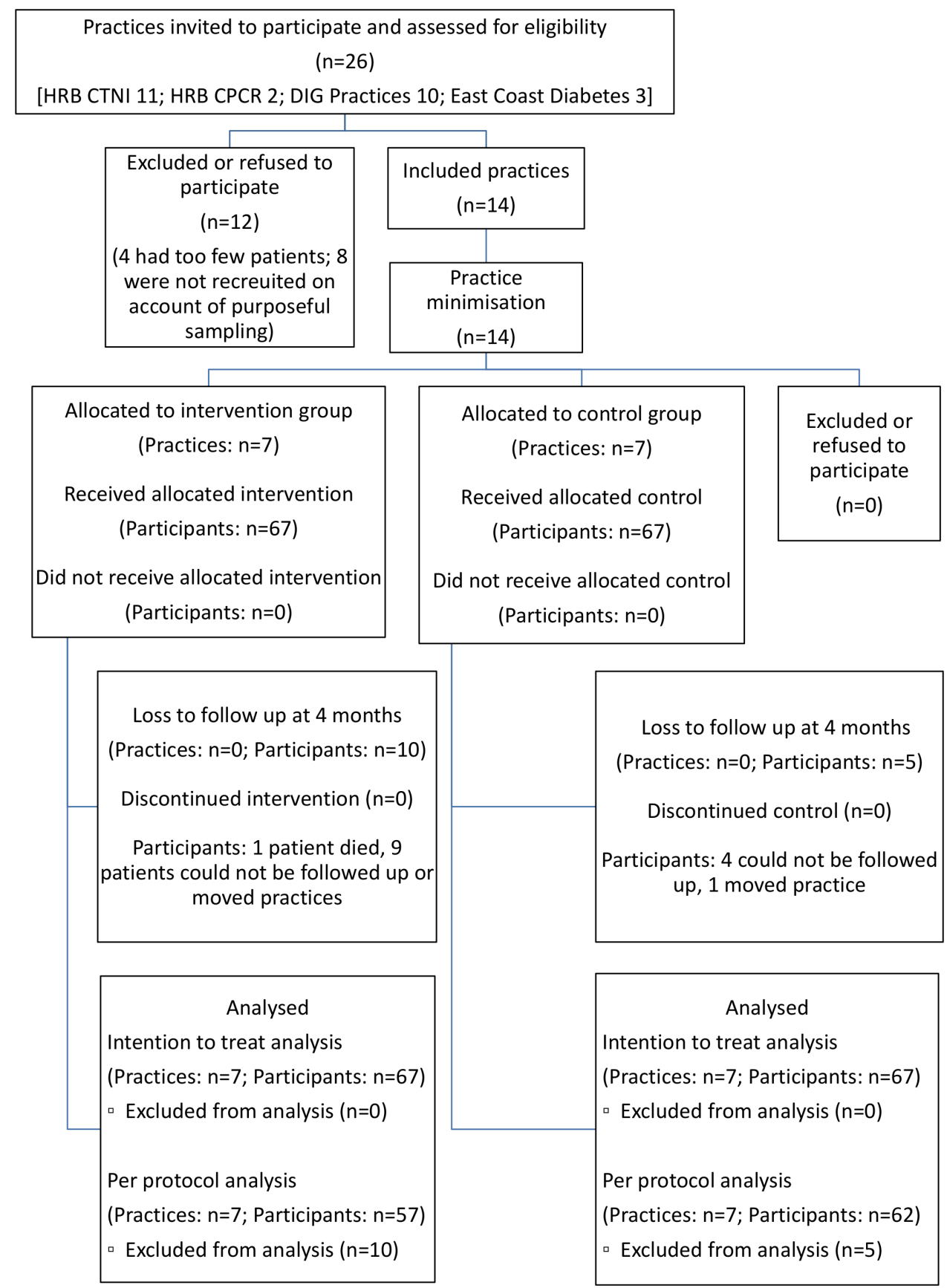

Figure 1 CONSORT flow diagram of practices and patients through the study. CONSORT, Consolidated Standards of Reporting Trials; DIG,Diabetes Interest Group; HRB CTNI, Health Research Board Clinical Trials Network Ireland; HRB CPCR, Health Research Board centre for primary care research. 
patients with a $\mathrm{HbA1c} \geq 70 \mathrm{mmol}$ and $\mathrm{BP} \geq 150 / 95 \mathrm{~mm} \mathrm{Hg}$ was 1.6 per practice, 11.9 patients per practice with a HbAlc $\geq 70 \mathrm{mmol}$ (and $\mathrm{BP}<150 / 95 \mathrm{~mm} \mathrm{Hg}$ ) and 6.4 patients per practice with a $\mathrm{BP} \geq 150 / 95 \mathrm{~mm} \mathrm{Hg}$.

All 14 practices and $119(88.8 \%)$ patients were followed up after 4 months, including $57(85.1 \%)$ interventionpractice patients and $62(92.5 \%)$ control-practice patients. Practices (cluster units) and patients were similar at baseline, except some differences in secondline anti-hyperglycaemic agents and numbers of patients with T2DM (see table 2).

\section{Feasibility and acceptability of the Decide intervention}

Analysis of the DECIDE-website CDSS-actions, highlighted how GPs considered and used recommended actions, both pharmacological and non-pharmacological (online supplementary file 7). Non-pharmacological actions $(n=210)$ were made more frequently than pharmacological-intensification actions $(n=117)$. The 210 non-pharmacological actions included 13 subgroups such as re-referral to the hospital setting/endocrinologist, acknowledging non-adherence with the patient, placing alerts in the patient's chart and contacting the patient by telephone to discuss medication intensification recommendations (online supplementary file 8).

Pharmacological intensification relating to antihyperglycaemic medications $(n=48)$ was more common than for anti-hypertensive $(\mathrm{n}=21)$ and lipid lowering medication ( $\mathrm{n}=49)$ (online supplementary file 7$)$. However, not all website actions were translated into changes in prescriptions when follow-up prescriptions were analysed and one GP did not record decisions made on the DECIDE website. Analysis of changes made to anti-hyperglycaemic medications indicated a range from 'no-intensification' coupled with a free text explanation $(n=25)$, to addition of a new medication $(n=15)$ and increasing dose of a medication $(n=8)$ (online supplementary file 9). GPs recorded a range of reasons for not intensifying medications including hesitancy around insulin pending hospital appointments $(\mathrm{n}=13)$, poor lifestyle behaviours or reduced adherence with medications $(\mathrm{n}=7)$, severe comorbid conditions (eg, cancer or severe psychiatric illness) $(\mathrm{n}=5)$ and recent improvements in control or awaiting response to a new medication $(n=5)$. Within anti-hypertensive lowering medications the addition of a new medication $(n=9)$ or switching of medication $(n=5)$ were the most common actions with no intensification in seven cases (online supplementary file 10).

Overall, the process evaluation indicated that DECIDE was acceptable to intervention GPs (see table 3). GPs liked the reminders about medication options and the opportunity to step back and consider a vulnerable patient group. The two main issues going forward relate to the type of patients targeted and the lack integration of CDSS recommendations into the EHR. Despite the fact that GPs generally welcomed the evidence-based recommendations, medication intensifications were modest. GPs reported that they were already aware of most of the patients identified, but suboptimal control wasn't simply related to lack of medication intensification. There were a broader range of barriers to good control including multimorbidity and non-engagement by patients with traditional GP and hospital services.

\section{Clinical outcomes}

At follow-up, there were no significant differences between intervention and control groups in relation to HbA1c, SBP, DBP, total cholesterol, medication intensification or utilisation of services (with the exception of one model for total cholesterol) and both groups had shown improvements over time (see table 4). Preplanned PP and subgroup analyses were also conducted and showed no differences between groups. It is uncertain why total cholesterol may have risen in the intervention group in one model, especially as there was no difference in the intensification of lipid-lowering agents between control and intervention practices.

\section{Health economic outcomes}

The process evaluation indicated that the resources identified, and the data collection methods used, were feasible and acceptable. At follow-up, there were no significant differences between intervention and control groups in relation to healthcare utilisation; thereby suggesting that the additional cost of implementing the intervention may not be offset in this short-term time horizon. A health economic evaluation is required to explore the value or cost effectiveness of the intervention. To this end, data collected on healthcare resource use would be supplemented by data collected via a preference-based generic health status instrument for the purposes of generating quality-adjusted life years, and a cost utility analysis conducted.

\section{DISCUSSION}

This pilot cluster RCT of DECIDE suggested that it is feasible for GPs but needs integration of the CDSS into the EHR and consideration of which patients to include. GPs valued the delivery of evidence-based medication intensification options at the point of care-delivery, in the context of increasingly complex guidelines and numbers of new anti-hyperglycaemic agents. While they also thought the focus on this cohort of patients was useful, they were sceptical about the role of newer medications for these patients and thought that patient-factors predominantly explained suboptimal control, such as non-engagement with healthcare services, reduced adherence with medications, multimorbidity and frailty. The intervention met preplanned continuation criteria to progress to a definitive RCT, but issues raised in the process evaluation would need to be addressed.

Strengths of the study include its pragmatic design, and high practice and patient retention, especially given difficulties GPs expressed with engagement with this cohort of patients. We followed the MRC framework and used the BCW to develop an intervention designed to carefully 
Table 2 Baseline characteristics of DECIDE practices and patients in intervention and control groups

\section{Characteristic}

Intervention practices

Control practices

Practice

$\mathrm{n}=7$

$\mathrm{n}=7$

Practice type

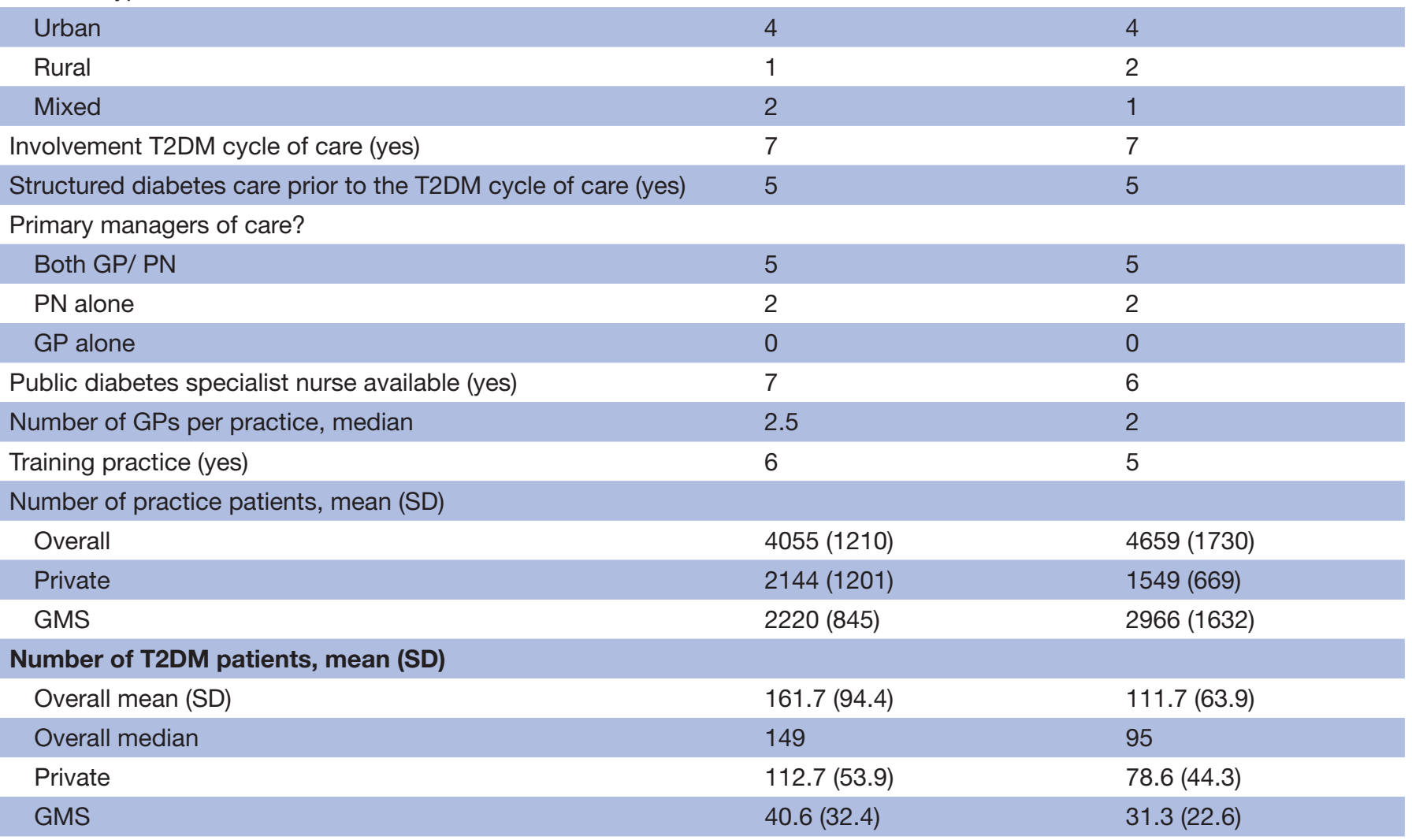

Electronic health record system

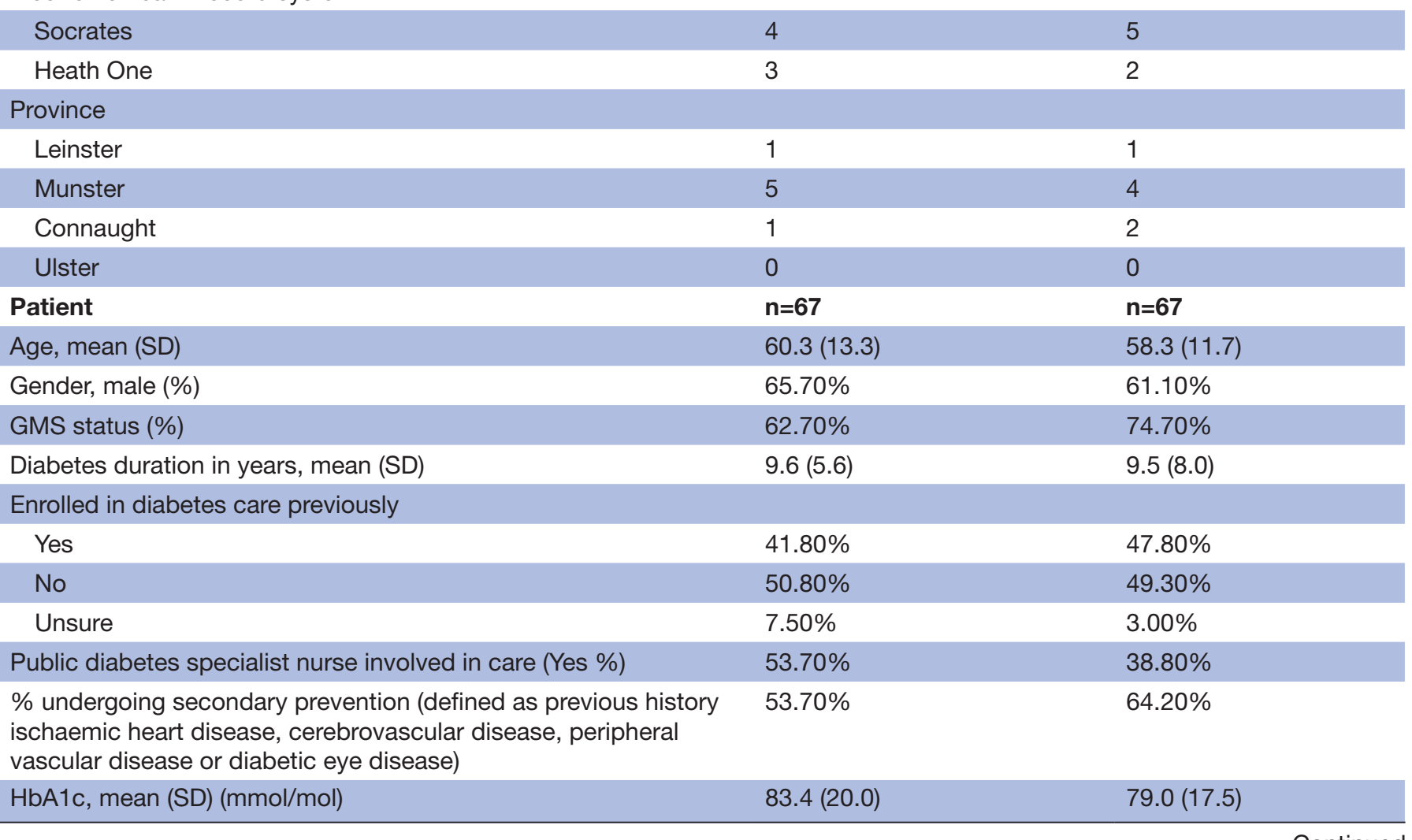

Continued 
Table 2 Continued

\begin{tabular}{|c|c|c|}
\hline Characteristic & Intervention practices & Control practices \\
\hline SBP, mean (SD) (mm Hg) & $135.7(20.7)$ & $133.6(15.7)$ \\
\hline DBP, mean (SD) (mm Hg) & $79.7(12.3)$ & $79.7(8.0)$ \\
\hline Total cholesterol, mean (SD); mmol/L & $4.7(1.6)$ & $4.8(2.0)$ \\
\hline Total medication number, median & 8 & 9 \\
\hline \multicolumn{3}{|l|}{ Hyperglycaemia lowering medications } \\
\hline Number glycaemic medications, median & 2 & 2 \\
\hline$\%$ taking glycaemic medications overall & $97.00 \%$ & $98.50 \%$ \\
\hline Metformin (\%) & $71.60 \%$ & $70.20 \%$ \\
\hline Sulphonylurea (\%) & $29.90 \%$ & $28.40 \%$ \\
\hline DPP-4 inhibitor (\%) & $37.30 \%$ & $25.40 \%$ \\
\hline GLP-1 agonist (\%) & $13.40 \%$ & $28.40 \%$ \\
\hline SGLT2 inhibitor (\%) & $17.90 \%$ & $26.90 \%$ \\
\hline Thiazolidinedione (\%) & $0.00 \%$ & $3.00 \%$ \\
\hline Insulin (\%) & $41.80 \%$ & $40.30 \%$ \\
\hline \multicolumn{3}{|l|}{ Anti-hypertensive medications } \\
\hline Number anti-hypertensive medications, median & 1 & 1 \\
\hline$\%$ taking hypertensive medications overall & $74.60 \%$ & $73.10 \%$ \\
\hline RAAS medication (\%) & $65.70 \%$ & $58.20 \%$ \\
\hline CCB (\%) & $29.90 \%$ & $23.90 \%$ \\
\hline Diuretic (\%) & $22.40 \%$ & $23.90 \%$ \\
\hline Beta-blocker (\%) & $26.90 \%$ & $25.40 \%$ \\
\hline Alpha-blocker (\%) & $0.00 \%$ & $3.00 \%$ \\
\hline Other $(\%)$ & $7.50 \%$ & $6.00 \%$ \\
\hline \multicolumn{3}{|l|}{ Lipid lowering medications } \\
\hline Number lipid lowering medications, mean (SD) & $0.9(0.6)$ & $0.8(0.5)$ \\
\hline$\%$ taking lipid lowering medications overall & $76.10 \%$ & $73.10 \%$ \\
\hline Statin (\%) & $68.70 \%$ & $73.10 \%$ \\
\hline Ezetimibe (\%) & $11.90 \%$ & $4.50 \%$ \\
\hline Fibrate (\%) & $1.50 \%$ & $1.50 \%$ \\
\hline
\end{tabular}

CCB, Calcium channel blocker; DBP, diastolic blood pressure; DECIDE, ComputeriseD dECislonal support for suboptimally controlleD typE 2 Diabetes mellitus in Irish General Practice; DPP-4, dipeptidyl peptidase 4; GLP-1, Glucagon-like peptide 1; GMS, General Medical Services; GP, general practitioner; HbA1c, glycated haemoglobin; PN, practice nurse; RAAS, renin-angioetnsion-aldotersone-system; SBP, systolic blood pressure; SGLT2, sodium-glucose transport protein 2; T2DM, type 2 diabetes mellitus.

address behaviour change in GPs. Many factors which support CDSS were incorporated into our intervention, including patient-specific advise, referenced evidencebased recommendations and being professionallyorientated ${ }^{47}$ Potential limitations related to the allocation of practices prior to baseline data collection but this was unavoidable, given that the website was used to identify patients and collect baseline data simultaneously. Integration of DECIDE into the EHR would have enabled easier workflow, as articulated by intervention GPs. ${ }^{47} 48$ Further limitations relate to the qualitative interviews being performed by one study team member, which potentially limited the range of feedback, though the data collected indicates this was not an issue with rich feedback from participants. The pilot nature of the study meant a short follow-up time which limited the ability to detect differences that could have resulted from medication intensification. In fact, $\mathrm{HbA1c}$ also fell in the control group from $79.0 \mathrm{mmol} / \mathrm{mol}$ to $70.8 \mathrm{mmol} / \mathrm{mol}$ with a $23 \%$ medication intensification rate. This likely occurred as patients in the control group were also identified by the finder tool, which would have alerted the GPs and enhanced care, mediated through medication changes. A future definitive RCT would need to address this issue. GPs reported that they performed this intervention mostly in their own time, so supporting this workload within the existing working day, in a sustainable manner would need to be factored in a future intervention. Finally, DECIDE 
Table 3 Feasibility and acceptability of the DECIDE intervention: benefits, barriers, feasibility and suggested modifications, from semi-structured interviews with intervention-GPs $(n=7)$

\section{Benefits of the DECIDE} intervention

\section{General positivity towards intervention:}

- "Overall the structure for me was very good. It was very well run." (GP04)

"I think the intention is right and the end points that we measure are important. No, I think it's good." (GP05)

"I certainly would do it again." (GP06)

\section{Evidence on DECIDE website was useful:}

- "... it was very, very helpful to have, you know on your website... to have kind of a flag coming up or saying "Look, this is the evidence for, and this is what should be done." (GP05)

- "It would prompt me, yes, it would prompt me. Not that I wouldn't do that anyway in most patients but yes, I would think that it would prompt me to actually have a good look and have a reason why l'm not doing it then." (GP06)

"I found it useful. I found some of the newer diabetic drugs can be a little bit tricky. You can get into a comfort zone where maybe you're prescribing a certain group of tablets and you can be a little bit reluctant to move from those." (GP09)

"The problem often is the number of diabetic medications has become a bit unwieldy.... it puts everything in front of you on a screen, which I don't always do. You tend to have these in your head but often when you actually sit down and look at it from that point of view." (GP10)

- "I thought even just itemising it and you had this visual, very clear visual representation of the meds they were on already." (GP11)

"It was very helpful to have a decision-making kind of tree to refer to"; "It was definitely useful to think and to be prompted to look at the evidence." (GP14)

But can be too prescriptive:

- "It does feel like a little bit like a prescription or recipe medicine." (GP05)

Educational material and the DECIDE folder was helpful:

- "I did like all the material in the side folder, even the little glimpses of medication." (GP06)

Supported engagement with patients:

- "They were very helpful, I thought. Absolutely, yeah. I suppose even to reinforce goals with the patients and stuff. I actually find that if you bring them in and if they are in front of you when you are doing it, they actually seem to engage an awful lot better. Generally speaking. They weren't in front of me for this but we started something else recently and being able to go through all of that with them, targeted, is very beneficial."

- "You are highlighting the patient in front of you. You are being forced into reviewing the notes more thoroughly and then I think it gives you options and even the lists of the different medication types when you are ticking the box and things like that. It's flagging straightaway, yeah, I need to look at this or we need to change there." (GP11)

"It certainly helped us to get focussed on some of our more difficult to manage patients." (GP14)

Finder Tool was helpful, highlighting patients who had 'gone through the cracks"

- "It brought it more into focus first of all, that the people who hadn't been looked at in a long time." (GP04)

- "To take up people who haven't been engaging and try and get them back engaging with us again." (GP04)

- "Maybe be a bit less fatalistic. You know, some of those patients certainly I thought - Look, he hasn't been in contact with us for more than a year, we certainly had tried. But yeah, I mean I thought - Look, yeah, we should give that guy a chance. I mean things might have changed, there might have been some personal circumstances that stopped him from engaging the last time we tried. Yeah. I think that certainly helped, yeah." (GP05)

- "But again, I found it really helpful to go and do that care review for all of these patients and it did pick up other things while I was doing that (laughter). For example, one of them we actually hadn't seen, she had fallen through the net, so that in itself I found very useful." (GP06)

- "Most of them wouldn't be bothered with hospital services as they are, they're seen very infrequently." (GP10)

- "it actually highlighted a couple of people who had kind of gone through the cracks a little bit, you know. Which was very helpful. There was probably three out of the ten, and one had actually moved practice." (GP11)

Extra discussion and enthusiasm

- "I think we actually had an extra discussion. We actually had a practice meeting about it and we actually had a discussion what we can improve ourselves on an ongoing basis So I think it was a good one to remind and refresh our team here that they stay enthusiastic about diabetes." (GP06)

- "and we actually had a discussion even only there 2 weeks ago, you know, what we would take away from it and what we think works and that's basically it in a nutshell. They felt it was a little bit - that the clientele that was chosen because they were uncontrolled already have a bias in them, because we would pride ourselves that the rest of them are excellently controlled. So, they took that as their professional pride that they weren't wel controlled." (GP06)

Continued 
Table 3 Continued

\section{Barriers to intervention delivery Issues with target population:}

- "Target of intervention (people with poor control) often don't engage, that's probably why their control is poor in the first place. So, in terms of getting results back to you, it was a bit of an effort to try and get some people in, to get their bloods done and also the blood pressure check, even to review them." (GP04)

- "The most common problem was really why they were kind of sub-optimally looked after really was because they weren't engaging." (GP05)

- "So maybe what it showed me was that a lot of the patients that are maybe suboptimally looked after either have a good valid reason for it, or are looked after by consultants and endocrine and don't want us to actually do anything. Or they are just not responding." (GP05)

- "I can see the benefit of a programme like DECIDE, it's just that for a vast majority of my patients, the reason why they weren't better controlled or there was no recent blood test or anything like that was frailty." (GP06)

- "They were all already our absolute heart sinks. I think it skews the results even a little bit for us because the rest of our diabetics are just well controlled because my nurses are doing a bloody good job. So, the ones that we were left with, they had good reasons to actually why they weren't controlled." (GP06)

"It's the fact that the tough ones are tough. They are probably tough for a reason. You know. The people who have poor control." (GP09)

"Find the main problem with managing diabetes is patients, having them in front of you, getting them to come back to you for their bloods." (GP10)

- "You can make in-roads into it, but I think incrementally you're making less and less in-roads you know, as you go for the tougher and tougher ones." (GP09)

"The more sicker patients or the ones that are more difficult to control, it is probably not as good with them but they, by their very nature are difficult to manage anyway, you know." (GP11)

- "Think a couple that we had in our group - they are so unwell at the moment that their care is at the other extreme... So we wouldn't end up changing his medications really. I think he had probably gone beyond the benefit of this." (GP11)

-We had a real problem getting the patients to come back. It is very difficult if you are dealing with a very different cohort of patients.... some of whom are very poorly controlled diabetics because of the fact that they are non-compliant or resisting treatment or change or simply just not attenders." (GP14)

- "We didn't have a lot of window in terms of actually making big impacts in terms of introducing new medications or even increasing doses. There were reasons for not doing that, whether side effects are just poly-pharmacy and patient resistance." (GP14)

Not that different from usual care:

- "From a practice level I wouldn't say it would have made a huge difference to us." (GP04)

- "We must have already tried fairly hard before this study to engage with the non-responders, so to say, and that we probably already have identified and dealt with patients where we can... that the people who were left over to be identified in this study, were actually patients where we couldn't do that much. Which was disappointing for the study, but maybe it means that actually GPs are already doing a lot of things right, maybe. I don't know." (GP05)

Limits to what DECIDE can achieve:

- "Yeah, I was a bit sad to see how... I would have thought yes, a bit more focus and a little bit more attention from us and me would make a big difference. But it didn't. And I thought 'Gosh, I'm a bit disappointed about that." (GP05)

-I do think medications can be a bit overdone in general diabetes because I don't have a huge amount of faith in a lot of them, in the newer ones, in that they all tend to bring your haemoglobin A1c down by about 1\%." (GP10)

-I suppose you could do everything perfectly and provide a really good service but all of those markers go up... So, I think the markers are important but some can miss really good care, you know." (GP11)

"The application of this outside of doing the one disease would be huge. Multi-morbidity is kind of the buzz word, isn't it now." (GP11)

Uncertainty of benefits due to time frame:

- "Trial was too short a time frame really to tell you whether you've got to have definite benefits from the intensification of the medication." (GP04)

Issues with DECIDE website:

" "Initial problems to actually log on." (GP06)

- "I was clicking something too quickly I couldn't go back then." (GP06)

"I would find that the little glitch there, that I had to ring you or email you back to make it active again when they were made inactive, but that is small fry!" (GP06)

- "It just needs to be an easy IT issue for the person, otherwise you'll find a lot of the people who are not that motivated or who are not IT savvy just won't use it." (GP09)

- "A practical problem at the start, I think I might have missed some of the screens early on.....might have been to do with the website itself or the way you had to scroll down through the bits." (GP11)

- "Yeah and then obviously just remembering where I put my log in and stuff. Those practical things." (GP11)

Continued 
Table 3 Continued

Feasibility of intervention

Not a team effort vs a team effort:

" "More than a nurse could do." (GP 04)

- "It wasn't really a team it was just me doing it...it would have been to laborious to actually educate everybody on it and then put a team together." (GP04)

- "The nurse was involved in ringing the patients up but basically she didn't know anything more about the study. Like I put all the data in. And it was no different for her chasing those patients up compared with previously." (GP05)

- "In our practice I did it all. But it didn't impact on them (other staff) at all." (GP11)

- "I think we have a highly motivated team, the girls are getting a kick out of having our diabetics well controlled. So I don't think there was an issue, no." (GP06)

- "I had all of my three nurses involved, all my three nurses involved in that. I think they were very much - it was a group effort." (GP06)

Patient distance:

- "Barriers as they always are, it is distance for us. Some of our patients live an hour away." (GP06)

Time required (completing outside normal hours):

- "I would have spent a lot of my own time, free time to get the study completed and that probably wouldn't be reflected in terms of the remuneration to the practice that we would have received for doing to study." (GP04)

- "Well you had to do it outside of normal working hours." (GP05)

- "Resource and time. Like I mean that's the one barrier that everybody has, I suppose." (GP05)

- "To protect the time to do it on an ongoing basis, you do it for a study and all of that, but you know what, that's life. That's nothing specific for the DECIDE study." (GP06)

- "I obviously didn't try and do it during my normal day or anything like that, I did it out of hours or out of my normal work. But I think if it was embedded or added into a consultation or stuff, I think it's easy enough but probably if its not embedded you would have to give yourself more time. Then you are looking at double entries and all of that. That just drives me mad." (GP11)

Acceptability to patients:

- "It was very acceptable. I don't think it impacted on the patients negatively." (GP06)

- "I suppose it made them aware of the option that we are there too to help...I think patients like to be asked." (GP05)

"I don't think they were probably aware of what happened really." (GP04)

- "I don't think there was actually any difference for them that they were actually called in and looked after and were given advice. They would be used to that." (GP06)

- "I think the patients love it. Anything like that, you know it's targeted, it's focussed, it's very concise and a clear consultation. I think they feel that they are really being looked after very well, you know. Again, it's an extra level of care almost, you know." (GP11)

Suggestions for intervention
"The data entry would have been quite onerous on it. I think possibly you could have sucked more data from the
patients file rather than having to manually enter it." (GP04)
"It would have been nice, obviously, if the website would have been able to communicate straight with Health
One." (GP05)
"It's like anything I suppose if you were trying to use it day to day, you'd like it embedded in your practice
software somehow." (GP11)
Longer time frame:
"But you probably need to leave a bit of a longer time frame. I think you probably will be looking at 6 to 12
months. By the time you get some reluctant customers in and patients in to get them rolling with it." (GP04)
Consider local differences in non-medication interventions:
"The non-medical kind of thing was too generic for my liking. I would have liked to have actually if you like free-
text." (GP06)

DECIDE, ComputeriseD dECislonal support for suboptimally controlleD typE 2 Diabetes mellitus in Irish General Practice; GP, general practitioner.

was designed as a cluster pilot RCT with an intervention targeting GPs and did not measure patient-reported outcome measures so we cannot comment on direct impact on patients' experiences. Discrepancies between the original trial registration and the pilot cluster RCT are discussed in online supplementary file 3 . We believe these changes were either unavoidable (including a changing data protection environment) or helped reduce bias. We did not have patients' body mass index available as an explanatory variable, which would have supported comparison of the groups in table 2 and provided context on what medications patients were prescribed, given the differing effects of anti-diabetic agents on weight.
While the follow-up length was short, in terms of effecting HbA1c, this was primarily a pilot study to assess feasibility and we also looked at medication intensification changes, which would be more immediate. Given that patient factors were such an important feature which GPs felt mediated significant elevation of blood pressure and hyperglycaemic, patient involvement in future research would also be essential. When 'recency of testing' was factored in the regression models, this reduced the number of patients significantly in the included model, which would need to be addressed in a definitive trial. Improving the recency of testing (including a blood pressure assessment and blood test) could happen with 


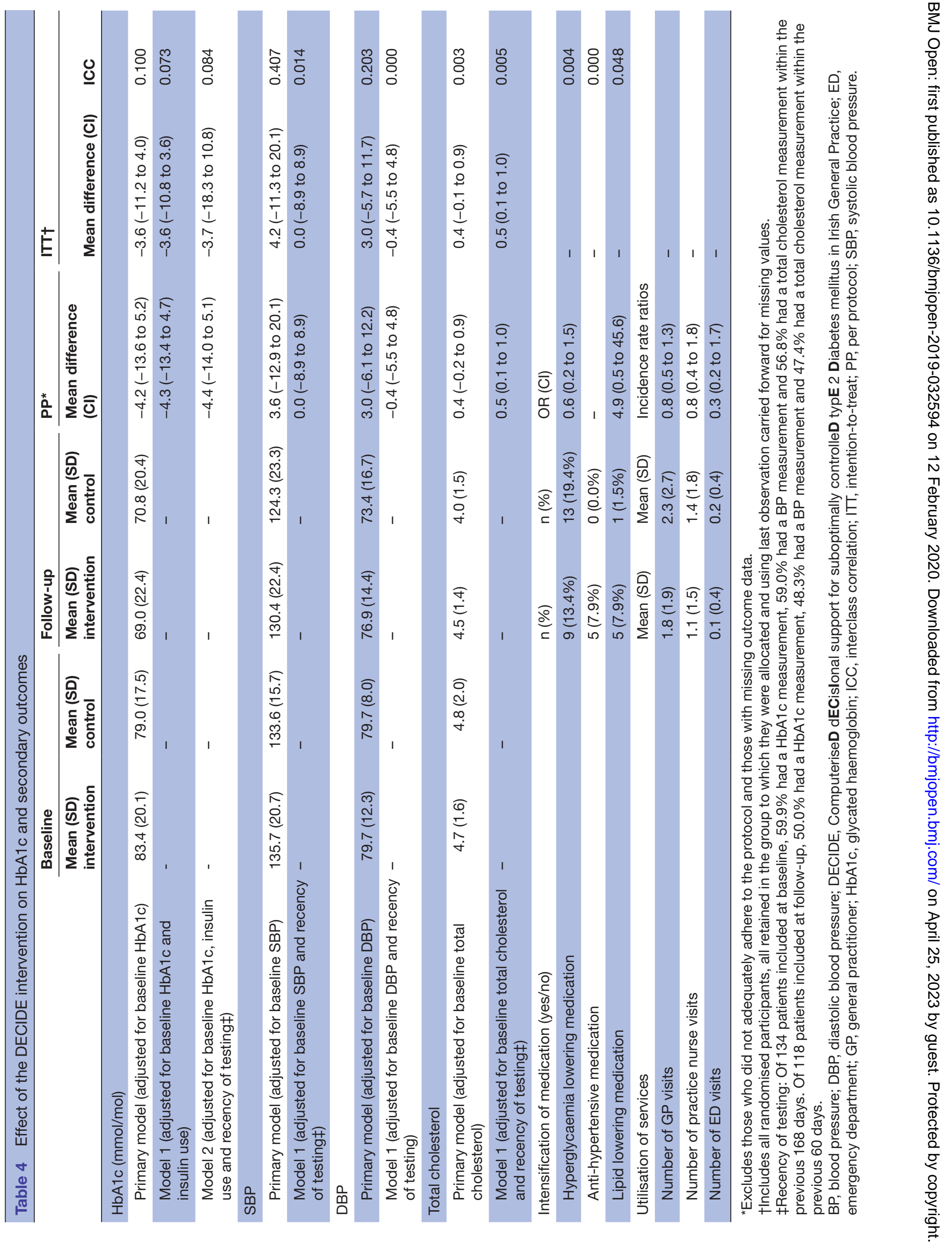


sufficient time, notice and resourcing of intervention and control practices.

All patients in our study had suboptimal control of their diabetes so had the potential for treatment escalation. The potential for treatment intensification for suboptimally controlled patients in primary care has been identified previously. ${ }^{18}$ In this previous study, GPs also reported hesitancy initiating insulin. Patients in our study were described as 'falling through cracks' and not attending or engaging in healthcare, despite robust practice procedures to call them back for review. When they did attend, some were perceived as difficult to manage or as 'heart break' patients. Parchman et al analysed 221 primary care encounters with patients with T2DM and found that each additional patient concern in the consultation, was associated with a $49 \%$ reduction in the likelihood of a change in medications, independent of the length of the encounter or the most recent HbAlc level. ${ }^{11}$ Sometimes only the most pressing issue can be addressed in a consultation, with symptomatic illness being of higher priority compared with surrogate outcomes like HbA1c. ${ }^{11}$ Patients can perceive poor control of T2DM as less important and diabetes can be relegated as an issue to be dealt with in the future. ${ }^{11}$ Parchman argued that clinical inertia, as a concept, does not fully characterise the complexity of primary care encounters and our study supports this concept. Some GPs in our study viewed suboptimal control as an acceptable and understandable phenomenon, given patient's comorbidities and complexities. This suggests that clinical inertia, as a hypothesis, should not be seen simply through a negative lens or viewed as medical conservatism. ${ }^{49}$

DECIDE was developed to address the gap in evidence for professionally-targeted interventions for suboptimally controlled T2DM and to target the behaviour and psychological capability of GPs who care for patients with poor control of T2DM. The previous professional intervention we had identified involved a decisional aid provided by UK-GPs to patients. ${ }^{50}$ It did not improve glycaemic control, though it did improve decisional conflict for patients. ${ }^{50}$ Our study had a different focus utilising a CDSS, aiming to modify GP behaviour. Previous reviews of CDSSs, in low risk patient groups, have shown limited impact on patient outcomes, but have improved processes of care. ${ }^{47}{ }^{51-53} \mathrm{In}$ DECIDE, if clinical outcomes were to improve, this would be partly mediated through intensification of medications. Our study suggests that delays in treatment escalation cannot be easily modified, however a definitive trial would be needed to determine this. However, there is limited evidence to date that CDSS can improve patient outcomes. ${ }^{47}$

Future research needs to address the likely impact on control practices if they are alerted about patients with suboptimal control. Electronic capture of control patient data without GP notification could address this and alternative designs such as stepped-wedge RCTs could be considered to allow sequential introduction of the IrishPrimary Care Research Network finder tool, and then the intervention to practices. ${ }^{54}$ Integration of the CDSS within the EHR should also be considered. ${ }^{47}$ A longer study, supporting medication intensification relating to insulin initiation, would be appropriate to capture this patient group who find it difficult to engage regularly with healthcare services. Also, the intervention may need to be broadened to support GPs managing diabetes in the context of comorbid disease, including psychiatric illness. Further research with patients can explore measures to support patients who have difficulties engaging in healthcare services. ${ }^{55-57}$

In this pilot cluster RCT, GPs welcomed decision support in the care of patients with suboptimally controlled T2DM, given the increasing complexity of diabetes management. There is clearly scope for CDSS interventions to support decision-making in this context, but interventions need to be integrated into the EHR and address the broader patient factors leading to poor control.

Twitter Mark E Murphy @drmarkmurphy and Jenny McSharry @jenmcsharry

Acknowledgements We would like to acknowledge the GPs and healthcare professionals who supported the pilot cluster randomised controlled trial and process evaluation, throughout Ireland. We would also like to thank colleagues and GPs who supported the intervention development process. The authors would also like to acknowledge support from the HRB-funded SPHeRE PhD Programme (Grant code: 1598), a research grant from the Irish College of General Practitioners, the WestREN Research Network and the HRB Primary Care Clinical Trials Network Ireland (Grant Code: CTN-2014-011).

Contributors All authors contributed to the drafting of this paper. MEM, SMS and TF conceived of the study idea. MB guided MEM through the Behaviour Change Wheel theoretical model. MB and JM supported theoretical development of the study and the process evaluation. MEM conducted the semi-structured interviews; MEM and JM analysed the qualitative data. MEM conducted the statistical analysis with support from FB and SMS. DC supported the development and maintenance of the DECIDE website and clinical decision support system. PG supported the pilot health economic evaluation.

Funding This work was supported by the Health Research Board (HRB) Centre for Primary Care Research grant number: HRC-2014-1.

Competing interests None declared.

Patient consent for publication Not required.

Ethics approval Ethical approval was granted by the Irish College of General Practitioners (ICGP).

Provenance and peer review Not commissioned; externally peer reviewed.

Data availability statement Data are available upon reasonable request. All data from the study (.dta and .xls files) can be shared upon reasonable request.

Open access This is an open access article distributed in accordance with the Creative Commons Attribution Non Commercial (CC BY-NC 4.0) license, which permits others to distribute, remix, adapt, build upon this work non-commercially, and license their derivative works on different terms, provided the original work is properly cited, appropriate credit is given, any changes made indicated, and the use is non-commercial. See: http://creativecommons.org/licenses/by-nc/4.0/.

\section{ORCID iDs}

Mark E Murphy http://orcid.org/0000-0002-4240-5327

Fiona Boland http://orcid.org/0000-0003-3228-0046

Tom Fahey http://orcid.org/0000-0002-5896-5783

\section{REFERENCES}

1 Stratton IM, Adler Al, Neil HA, et al. Association of glycaemia with macrovascular and microvascular complications of type 2 diabetes (UKPDS 35): prospective observational study. BMJ 2000;321:405-12.

2 Nolan JJ, O'Halloran D, McKenna TJ, et al. The cost of treating type 2 diabetes (CODEIRE). Ir Med J 2006;99:307-10.

3 Khunti K, Wolden ML, Thorsted BL, et al. Clinical inertia in people with type 2 diabetes: a retrospective cohort study of more than 80,000 people. Diabetes Care 2013;36:3411-7. 
4 Spann SJ, Nutting PA, Galliher JM, et al. Management of type 2 diabetes in the primary care setting: a practice-based research network study. Ann Fam Med 2006;4:23-31.

5 Campbell DJ, McGrady M, Prior DL, et al. Most individuals with treated blood pressures above target receive only one or two antihypertensive drug classes. Intern Med J 2013;43:137-43.

6 Mahase $\mathrm{E}$. Half of patients prescribed statins fail to hit target reduction in LDL cholesterol. BMJ 2019;365:I1838.

7 Murphy M, Galvin R, Boland F, et al. Effectiveness of interventions in primary care to improve glycated haemoglobin $(\mathrm{HbA1c})$ and cardiovascular risk factor levels in patients with poorly-controlled type 2 diabetes mellitus: a systematic review. PROSPERO 2017;8.

8 Khan H, Lasker SS, Chowdhury TA. Exploring reasons for very poor glycaemic control in patients with type 2 diabetes. Prim Care Diabetes 2011;5:251-5.

9 Reach G. Clinical inertia, uncertainty and individualized guidelines. Diabetes Metab 2014;40:241-5.

10 Lin J, Zhou S, Pan C, et al. Predictors of clinical inertia in a U.S managed care setting. Diabetes 2014;63:A308.

11 Parchman ML, Pugh JA, Romero RL, et al. Competing demands or clinical inertia: the case of elevated glycosylated hemoglobin. Ann Fam Med 2007;5:196-201.

12 Zafar A, Mehta R, Davies MJ, et al. Clinical inertia: failure to intensify the therapy when needed in the management of type 2 diabetes, retrospective analysis of Wandsworth (general practice) data (19982007). Diabetic Med 2011;28:198.

13 Zafar A, Davies M, Azhar A, et al. Clinical inertia in management of T2DM. Prim Care Diabetes 2010;4:203-7.

14 Balkau B, Bouée S, Avignon A, et al. Type 2 diabetes treatment intensification in general practice in France in 2008-2009: the DIAttitude study. Diabetes Metab 2012;38(Suppl 3):S29-35.

15 González-Clemente JM, Font B, Lahoz R, et al. [INERTIA study: Clinical inertia in non-insulinized patients on oral hypoglycemic treatment. A study in Spanish primary and specialty care settings]. Med Clin 2014;142:478-84.

16 Gil-Guillén V, Orozco-Beltrán D, Carratalá-Munuera C, et al. Clinica inertia in poorly controlled elderly hypertensive patients: a crosssectional study in Spanish physicians to ascertain reasons for not intensifying treatment. Am J Cardiovasc Drugs 2013;13:213-9.

17 Bralić Lang V, Bergman Marković B, Kranjčević K. Family physician clinical inertia in glycemic control among patients with type 2 diabetes. Med Sci Monit 2015;21:403-11.

18 Khunti K, Gomes MB, Pocock S, et al. Therapeutic inertia in the treatment of hyperglycaemia in patients with type 2 diabetes: a systematic review. Diabetes Obes Metab 2018;20:427-37.

19 Reach G, Pechtner V, Gentilella R, et al. Clinical inertia and its impact on treatment intensification in people with type 2 diabetes mellitus. Diabetes Metab 2017;43:501-11.

20 Bailey CJ. Under-treatment of type 2 diabetes: causes and outcomes of clinical inertia. Int J Clin Pract 2016;70:988-95.

21 Murphy ME, Fahey T, Smith SM. Computer-based clinical decision support for general practitioners. Fam Pract 2014;31:497-8.

22 Montori VM. Selecting the right drug treatment for adults with type 2 diabetes. BMJ 2016;352:i1663.

23 NICE Pathways. Blood-glucose-lowering therapy for type 2 diabetes, 2016.

24 Irish College of General Practitioners. A practical guide to integrated type 2 diabetes care, 2016.

25 Murphy ME, Byrne M, Zarabzadeh A, et al. Development of a complex intervention to promote appropriate prescribing and medication intensification in poorly controlled type 2 diabetes mellitus in Irish general practice. Implement Sci 2017;12:115.

26 Craig P, Dieppe P, Macintyre S, et al. Developing and evaluating complex interventions: the new medical Research Council guidance. BMJ 2008;337:a1655.

27 Michie S, Atkins L, West R. The behaviour change wheel. A guide to designing interventions. 1 edn. Silverback Publishing, 2014

28 Michie S, van Stralen MM, West R. The behaviour change wheel: a new method for characterising and designing behaviour change interventions. Implement Sci 2011;6:42

29 Murphy ME, Byrne M, Boland F, et al. Supporting General practitioner-based care for poorly controlled type 2 diabetes mellitus (the DECIDE study): feasibility study and protocol for a pilot cluster randomised controlled trial. Pilot Feasibility Stud 2018;4:159.

30 Eldridge SM, Chan CL, Campbell MJ, et al. CONSORT 2010 statement: extension to randomised pilot and feasibility trials. BMJ 2016;355:i5239.

31 Speight J, Conn J, Dunning T, et al. Diabetes Australia position statement. A new language for diabetes: improving communications with and about people with diabetes. Diabetes Res Clin Pract 2012;97:425-31.

32 Stamler J, Vaccaro O, Neaton JD, et al. Diabetes, other risk factors, and 12-yr cardiovascular mortality for men screened in the multiple risk factor intervention trial. Diabetes Care 1993;16:434-44.

33 Cushman WC, Evans GW, Byington RP, et al. Effects of intensive blood-pressure control in type 2 diabetes mellitus. $N$ Engl J Med 2010;362:1575-85.

34 Ginsberg HN, Elam MB, Lovato LC, et al. Effects of combination lipid therapy in type 2 diabetes mellitus. N Engl J Med 2010;362:1563-74.

35 Giorgino F, Leonardini A, Laviola L. Cardiovascular disease and glycemic control in type 2 diabetes: now that the dust is settling from large clinical trials. Ann N Y Acad Sci 2013;1281:36-50.

36 Patel A, MacMahon S, Chalmers J, et al. Intensive blood glucose control and vascular outcomes in patients with type 2 diabetes. $N$ Engl J Med 2008;358:2560-72.

37 Gerstein HC, Miller ME, Byington RP, et al. Effects of intensive glucose lowering in type 2 diabetes. N Engl J Med 2008;358:2545-59.

38 Duckworth W, Abraira C, Moritz T, et al. Glucose control and vascular complications in veterans with type 2 diabetes. $N$ Engl J Med 2009;360:129-39.

39 Saghaei M. An overview of randomization and minimization programs for randomized clinical trials. J Med Signals Sens 2011;1:55-61.

40 Michie S, Richardson M, Johnston M, et al. The behavior change technique taxonomy (V1) of 93 hierarchically clustered techniques: building an international consensus for the reporting of behavior change interventions. Ann Behav Med 2013;46:81-95.

41 Hoffmann TC, Glasziou PP, Boutron I, et al. Better reporting of interventions: template for intervention description and replication (TIDieR) checklist and guide. BMJ 2014;348:g1687.

42 Avery KNL, Williamson PR, Gamble C, et al. Informing efficient randomised controlled trials: exploration of challenges in developing progression criteria for internal pilot studies. BMJ Open 2017;7:e013537.

43 Hsieh H-F, Shannon SE. Three approaches to qualitative content analysis. Qual Health Res 2005;15:1277-88.

44 Thabane L, Hopewell S, Lancaster GA, et al. Methods and processes for development of a CONSORT extension for reporting pilot randomized controlled trials. Pilot Feasibility Stud 2016;2:25.

45 Schulz KF, Altman DG, Moher D, et al. Consort 2010 changes and testing blindness in RCTs. Lancet 2010;375:1144-6.

46 StataCorp. Stata statistical software: release 15. College Station, TX: StataCorp LP, 2018.

47 Van de Velde S, Heselmans A, Delvaux N, et al. A systematic review of trials evaluating success factors of interventions with computerised clinical decision support. Implement Sci 2018;13:114.

48 Kawamoto K, Houlihan CA, Balas EA, et al. Improving clinical practice using clinical decision support systems: a systematic review of trials to identify features critical to success. BMJ 2005;330:765.

49 Houston $\mathrm{M}$. When a tincture of time is better than a prescription, 2013. Available: http://www.irishtimes.com/life-and-style/health-family/whena-tincture-of-time-is-better-than-a-prescription-1.1464453

50 Mathers $\mathrm{N}, \mathrm{Ng} \mathrm{CJ}$, Campbell MJ, et al. Clinical effectiveness of a patient decision aid to improve decision quality and glycaemic control in people with diabetes making treatment choices: a cluster randomised controlled trial (PANDAS) in general practice. BMJ Open 2012;2:e001469.

51 Cleveringa FGW, Gorter KJ, van den Donk M, et al. Computerized decision support systems in primary care for type 2 diabetes patients only improve patients' outcomes when combined with feedback on performance and case management: a systematic review. Diabetes Technol Ther 2013;15:180-92.

52 Jeffery R, Iserman E, Haynes RB, et al. Can computerized clinical decision support systems improve diabetes management? A systematic review and meta-analysis. Diabet Med 2013;30:739-45.

53 Krass I, Schieback P, Dhippayom T. Adherence to diabetes medication: a systematic review. Diabet Med 2015;32:725-37

54 Hemming K, Haines TP, Chilton PJ, et al. The stepped wedge cluster randomised trial: rationale, design, analysis, and reporting. BMJ 2015;350:h391.

55 Crowley MJ, Holleman R, Klamerus ML, et al. Factors associated with persistent poorly controlled diabetes mellitus: clues to improving management in patients with resistant poor control. Chronic IIIn 2014;10:291-302.

56 Semenkovich K, Brown ME, Svrakic DM, et al. Depression in type 2 diabetes mellitus: prevalence, impact, and treatment. Drugs 2015;75:577-87

57 Bombard Y, Baker GR, Orlando E, et al. Engaging patients to improve quality of care: a systematic review. Implement Sci 2018;13:98. 